\title{
QUANTUM-THERMAL FLUCTUATIONS OF EFFECTIVE MACROPARAMETERS AND THEIR CORRELATIONS
}

\author{
A.D. SUKHANOV ${ }^{1}$ O.N. GOLUBJEVA, ${ }^{2}$ V.G. BARYAKHTAR ${ }^{3}$ \\ ${ }^{1}$ N.N. Bogoliubov Laboratory of theoretical physics, JINR \\ (Dubna, Russia; e-mail: ogol@mail.ru) \\ ${ }^{2}$ Peoples' Friendship University of Russia \\ (Moscow, Russia; e-mail: ogol@oldi.ru) \\ ${ }^{3}$ Institute of Magnetism, Nat. Acad. of Sci. of Ukraine \\ (Kyiv, Ukraine; e-mail: bvg@mail.vtv. kiev.ua)
}

PACS 05.70.-a, 05.20-y

(C) 2011

The application of the conventional theory of macroparameter fluctuations has been shown to go beyond the framework of the thermodynamic description in a number of cases. The principles of the theory of quantum-thermal fluctuations of effective macroparameters and their correlations have been formulated. The theory satisfies the applicability conditions of equilibrium thermodynamics and is based on effective macroparameters, which take the integral stochastic action of the environment into account at any temperatures. The correlator of conjugate macroparameters, namely, the effective entropy and the effective temperature, has been calculated. The correlator was found to be proportional to the effective action which characterizes the stochastic environment. The pair correlators for the conjugate effective parameters "entropy-temperature" and "coordinate-momentum" have been demonstrated to depend linearly on the effective action, with their minimum values being determined by Planck's constant.

\section{Problem of Accounting for Quantum Effects in the Macroparameter Fluctuation Theory}

In the last years, the necessity in the application of thermodynamics to relatively small objects (nanoparticles, nuclear spins, and so forth), which are in the thermal equilibrium at low temperatures, has been recognized. Thus, a necessity to attentively analyze the fundamentals of thermodynamics arose.

The equilibrium thermodynamics is known to be based upon four principles (laws). Among them, the zeroth law, which introduces the fundamental idea of thermal equilibrium between an object and its macroenvironment (called a thermostat), is primordial. In the classical thermodynamics, all macroparameters are determined rigorously, so that the zeroth law looks like a condition for the "strict equality" between the temperatures of the object, $T$, and the thermostat, $T_{0}$,

$T=T_{0}$
This relation is equivalent to understanding the temperature, which is measured on the Kelvin scale, as a conditional "marker" for the thermal equilibrium.

In statistical thermodynamics [1-5], including the macroparameter fluctuation theory, every macroparameter $A$ of the object, including the temperature, is regarded as a random variable characterized by fluctuations $\delta A$ around its average value $\langle A\rangle$. To preserve the terminology used in the traditional thermodynamic description, the additional requirement is put forward simultaneously, namely, the relative dispersion of an arbitrary macroparameter $A$ is confined by the condition ${ }^{1}$

$\frac{D(A)}{\langle A\rangle^{2}} \leqslant 1$

Here,

$D(A) \equiv\left\langle(\delta A)^{2}\right\rangle=\left\langle A^{2}\right\rangle-\langle A\rangle^{2}$

is the dispersion of macroparameter $A$ calculated by averaging over a distribution typical of the macroparameter fluctuation theory [1-3]. Below, for convenience, we use the standard deviation $\Delta A=\sqrt{D(A)}$.

In this case, the concept of thermal equilibrium obtains a generalized meaning. It is understood that the object temperature can also fluctuate (a "soft" equilibrium condition) owing to the thermal stochastic action by the thermostat, which is characterized by the Boltzmann constant $k_{\mathrm{B}}$. This means that a requirement similar to inequality (2) is imposed on the magnitude of $\Delta T$ as well. At the same time, the temperature of the thermostat, as a system with the infinite number of degrees of freedom, does not fluctuate, i.e. $\Delta T_{0}=0$.

\footnotetext{
${ }^{1}$ In the cases where the denominator in Eq. (2) is nullified, Bogoliubov's quasiaverage [6] rather than Gibbs's average has to be used.
} 
As a result, the zeroth law in the statistical thermodynamics is expressed as a collection of two conditions,

$T=T_{0} \pm \Delta T ; \quad \frac{(\Delta T)^{2}}{T_{0}^{2}} \leqslant 1$,

where $\Delta T$ is the standard deviation from $\langle T\rangle$, and $(\Delta T)^{2}$, according to the notations in formula $(2)$, is the temperature dispersion. Hence, only the average temperature of the object, $\langle T\rangle$, coincides now with the temperature of thermostat, $T_{0}$.

As is known, in the standard fluctuation theory [2,3], expressions for dispersions of arbitrary macroparameters can be obtained on the basis of the distribution with the modulus $\Theta$. Involving phenomenological results, the quantity $\Theta$ is usually written down in the form $\Theta=k_{\mathrm{B}} T_{0}$. We attract attention to the fact that this expression implies that a model consisting of a collection of classical oscillators is implicitly used for the thermostat. Below, we refer to it as the classical model.

In particular, A. Einstein also found formulas [1] for the temperature dispersion,

$$
(\Delta T)^{2}=\frac{1}{k_{\mathrm{B}} C_{V}} \Theta^{2}=\frac{k_{\mathrm{B}}}{C_{V}} T_{0}^{2},
$$

and the dispersions of the object's internal energy $U$ at a constant volume $V$,

$$
(\Delta U)^{2}=\frac{C_{V}}{k_{\mathrm{B}}} \Theta^{2}=k_{\mathrm{B}} C_{V} T_{0}^{2},
$$

where

$$
C_{V}(T, V)=\left.\frac{\partial U}{\partial T}\right|_{V}
$$

is the heat capacity of the object.

It is of interest to elucidate which are the objects, for which the expression obtained above satisfies the inequality in condition (2). The answer is contained in a specific expression for the heat capacity. For instance, for the macroscopic objects consisting of atoms, $U \sim N$ and $\left(C_{V}\right) \sim N$, where $N$ has an order of the Avogadro number. Hence, the dispersion $(\Delta T)^{2} \sim \frac{1}{N}$. Accordingly, $(\Delta U)^{2} \sim N$. Therefore, condition (2) is satisfied for the relative dispersions of internal energy and temperature in such objects at high temperatures, if $N \gg 1$.

At the same time, we attract attention to the fact that, for example, in the case of a single classical oscillator $(N=1)$, for which $U=k_{\mathrm{B}} T$ and $C_{V}=k_{\mathrm{B}}$, and which is in equilibrium with the thermostat, we have

$$
\frac{(\Delta U)^{2}}{\langle U\rangle^{2}}=1 ; \quad \frac{(\Delta T)^{2}}{\langle T\rangle^{2}}=1 \text {. }
$$

Thus, the condition of macroscopicity $(N \gg 1)$ is not strictly mandatory, while carrying out calculations in the framework of classical statistical mechanics and in the high temperature range.

A different situation arises at relatively low temperatures, when quantum-mechanical effects manifest themselves. In this case, the main attention is still given to the heat capacity

$$
\left(C_{V}\right)_{\mathrm{qu}}=\left.\frac{\partial U_{\mathrm{qu}}}{\partial T}\right|_{V}
$$

but, for its calculation, the internal energy $U_{\mathrm{qu}}$ is applied, which is calculated now in the framework of the quantum statistical mechanics. At the same time, the model of thermostat does not undergo changes and remains classical. On this route, the satisfactory results can still be obtained for systems of particles with $N \gg 1$ (except for, maybe, the region of ultralow temperatures). However, there emerge the problems for the equilibrium thermal radiation (where the concept of the number of particles is absent) and a single quantum-mechanical oscillator.

Let us demonstrate the aforesaid using the quantummechanical oscillator as an example. Its internal energy is equal, according to A. Einstein [7], to

$U_{\mathrm{qu}}=\frac{\hbar \omega}{\exp \left\{2 \varkappa \frac{\omega}{T}\right\}-1}=\frac{\hbar \omega}{2} \frac{\exp \left\{-\varkappa \frac{\omega}{T}\right\}}{\sinh \left(\varkappa \frac{\omega}{T}\right)}$,

and the heat capacity is

$\left(C_{V}\right)_{\mathrm{qu}}=k_{\mathrm{B}}\left(\frac{\hbar \omega}{k_{\mathrm{B}} T}\right)^{2} \frac{\exp \left\{2 \varkappa \frac{\omega}{T}\right\}}{\left(\exp \left\{2 \varkappa \frac{\omega}{T}\right\}-1\right)^{2}}=$

$=k_{\mathrm{B}}\left(\varkappa \frac{\omega}{T}\right)^{2} \frac{1}{\sinh ^{2}\left(\varkappa \frac{\omega}{T}\right)}$,

where the notation

$\varkappa=\hbar / 2 k_{\mathrm{B}}$,

was introduced. In accordance with the general formula (5), the dispersion of the internal energy of a quantummechanical oscillator at the replacement of $\left(C_{V}\right)$ by $\left(C_{V}\right)_{\text {qu }}$ looks like

$$
\begin{aligned}
& \left(\Delta U_{\mathrm{qu}}\right)^{2}=k_{\mathrm{B}}\left(C_{V}\right)_{\mathrm{qu}} T^{2}=\left(\frac{\hbar \omega}{2}\right)^{2} \frac{1}{\sinh ^{2}\left(\varkappa \frac{\omega}{T}\right)}= \\
& =\hbar \omega U_{\mathrm{qu}}+U_{\mathrm{qu}}^{2}=\exp \left\{2 \varkappa \frac{\omega}{T}\right\} U_{\mathrm{qu}}^{2},
\end{aligned}
$$


and the relative dispersion of its internal energy reads

$$
\frac{\left(\Delta U_{\mathrm{qu}}\right)^{2}}{U_{\mathrm{qu}}^{2}}=\frac{\hbar \omega}{U_{\mathrm{qu}}}+1=\exp \left\{2 \varkappa \frac{\omega}{T}\right\} .
$$

A similar result is obtained for the relative dispersion of the thermal radiation energy in the spectral interval $(\omega, \omega+\Delta \omega)$ in the volume $V$,

$$
\frac{\left(\Delta U_{\omega}\right)^{2}}{U_{\omega}^{2}}=\frac{\hbar \omega}{U_{\omega}}+\frac{\pi^{2} c^{3}}{V \omega^{2} \Delta \omega}=\frac{\pi^{2} c^{3}}{V \omega^{2} \Delta \omega} \exp \left\{2 \varkappa \frac{\omega}{T}\right\} .
$$

Those facts evidently testify to the inapplicability of the selected method of calculation; namely, the relative dispersions of internal energy (9) and (10) do not satisfy condition (2) for the thermodynamic description to be applicable. Note that this circumstance did not draw a special attention of researchers. A.I. Anselm [8] was probably the only who marked it. In this connection, he pointed out that "the conventional thermodynamics is not applicable as the temperature falls down". This means that the statistical thermodynamics considered as a macrotheory cannot be based exclusively upon the quantum statistical mechanics as a microtheory.

In our opinion, all that originates from the fundamental credo of quantum statistical mechanics, according to which the quantum-mechanical and thermal actions of the environment can be taken into consideration independently. In this connection, the standard routine consists in that the quantum-mechanical characteristics of a system of microobjects are determined firstly (at $T_{0}=0$ ), and only then the system is embedded into a classical thermostat with the distribution modulus $k_{\mathrm{B}} T_{0}$. At the same time, formula (6) for a quantum-mechanical oscillator and an analogous formula for the internal energy of the thermal irradiation, which are confirmed by experiment, testify that those two types of stochastic action manifest themselves, as a rule, jointly and nonadditively.

In order to overcome the arisen problems, we developed a theory [8-11], which is based on a combined account of the quantum-mechanical and thermal stochastic actions by the environment. The theory denies the classical model of thermostat in favor of the quantum-mechanical one, including the case of heat capacity calculations. Moreover, in this case, the introduction of a different "marker" for the thermal equilibrium state is required. The matter is that the Kelvin temperature used for such purposes in the quantum statistical mechanics turns out noninformative in the region, where the quantum-mechanical and thermal effects jointly manifest themselves, because this characteristic reflects only the thermal stochastic action (in terms of the Boltzmann constant). In addition, it is adopted to be equal to zero in quantum mechanics, where, nevertheless, the stochastic action (although being already of the quantum-mechanical type in terms of Planck's constant) takes place by essence.

Purely intuitive considerations stimulate us to adopt the expression that follows from the Planck formula for the energy of quantum-mechanical oscillator $U_{\mathrm{Pl}}$, as a new "marker"; namely,

$$
\mathbb{T} \equiv \frac{U_{\mathrm{Pl}}}{k_{\mathrm{B}}}=\frac{\hbar \omega}{2 k_{\mathrm{B}}} \operatorname{coth} \frac{\hbar \omega}{2 k_{\mathrm{B}} T}=\varkappa \omega \operatorname{coth} \frac{\varkappa \omega}{T},
$$

where the notation $\varkappa=\hbar / 2 k_{\mathrm{B}}$ introduced earlier is used. We define this quantity as the effective temperature. It is important that, at the Kelvin temperature $T=0$, the effective temperature has a nonzero minimum,

$\mathbb{T}^{\min }=\varkappa \omega \neq 0$.

In the general case, according to formulas (11) and (12), the effective temperature is a two-parameter function $\mathbb{T}=f(T, \omega)$. It can be reduced to a one-parameter function only in the limiting cases $T=0$ and $T \gg \mathbb{T}^{\mathrm{min}}$, i.e., where $\mathbb{T} \approx \mathbb{T}^{\min }$ and $\mathbb{T} \approx T$, respectively. Its simultaneous dependence on the Planck and Boltzmann constants allows one to assert that, in view of the relation $\varkappa=\frac{\hbar}{2 k_{\mathrm{B}}}$, the stochastic action of the "thermal" type cannot be ignored even at the Kelvin temperature $T_{0}=0$

On this basis, we constructed a theory of fluctuations for macroparameters and their correlations, which is presented below. In so doing and keeping preserved all traditional relations between thermodynamic quantities coupled with the temperature, we introduced the corresponding effective macroparameters, which are the same functions of the effective temperature.

\section{Effective Temperature and Effective Internal Energy Fluctuations}

To calculate the dispersions of macroparameters taking the stochastic action of the quantum-mechanical type into account at any temperatures, let us use the results of work [9], which is a macrodescription in the framework of a theory called by us the modern stochastic thermodynamics [11]. It is based on the Gibbs distribution in the space of macroparameters $[5,12]$.

To describe the stochastic environment, we introduce a quantum-mechanical model (a quantum thermostat) that combines the concepts of vacuum and thermostat. 
It comprises an infinite set of quantum-mechanical normal modes of all frequencies at any fixed Kelvin temperature, being an analog of equilibrium thermal radiation. The effective temperature of the object (11) is supposed to fluctuate as well with a characteristic frequency $\omega$, so that the zeroth law reads

$\mathbb{T}=\mathbb{T}_{0} \pm \Delta \mathbb{T}$

in the developed theory, where $\mathbb{T}_{0}$ is the effective temperature of the thermostat, and $\Delta \mathbb{T}$ is the standard deviation of the effective temperature of the object under the equilibrium conditions.

From the modern viewpoint, the primordial principle of the fluctuation theory of macroparameters is the principle of entropy maximum in the thermal equilibrium state. The exponential Gibbs form for the distribution in the space of macroparameters follows from it [12],

$d W(\mathcal{E})=\rho(\mathcal{E}) d \mathcal{E}=\frac{1}{\Theta} \exp \left\{-\frac{\mathcal{E}}{\Theta}\right\} d \mathcal{E}$.

Here, $\mathcal{E}$ is a random value of the internal energy of a macroobject, which depends on the macroparameters that characterize the type of its interaction with the environment.

One should bear in mind that, in the initial version of the macroparameter fluctuation theory, the distribution modulus corresponded to the classical model of thermostat with the Kelvin temperature $T_{0}$. Since the developed theory uses the quantum-mechanical model of thermostat, the corresponding distribution modulus is expressed in terms of the effective thermostat temperature $\mathbb{T}_{0}$ like $k_{\mathrm{B}} \mathbb{T}_{0}$. As a result, the standard formula for the macroparameter fluctuation distribution [1-3] and the expressions for the dispersions of any macroparameters, which follow from it, remain formally invariable, but they are expressed now in terms of effective macroparameters. Therefore, the dispersion of the effective temperature of a macroobject, instead of formula (4), looks like

$(\Delta \mathbb{T})^{2}=\frac{k_{\mathrm{B}}}{\mathbb{C}_{V}} \mathbb{T}_{0}^{2}$

$\mathbb{C}_{V} \equiv \frac{\partial \mathbb{U}}{\partial \mathbb{T}}$

is the effective heat capacity of the object. Accordingly, for the dispersion of the effective internal energy, instead of formula (5), we obtain

$(\Delta \mathbb{U})^{2}=k_{\mathrm{B}} \mathbb{C}_{V} \mathbb{T}_{0}^{2}$.
In other words, all expressions (13)-(17) preserve their earlier forms in the new theory. In this way, the concept of the equilibrium state between an object and a stochastic environment remains also invariable. However, this equilibrium is now a generalized concept, which involves the existence of two nonadditive types of stochastic action in the integral concept of effective temperature $\mathbb{T}$.

Now, let us apply the results obtained to the study of a macroobject that can be represented as a quantummechanical oscillator $^{2}$. For this object, $\mathbb{U} \equiv U_{\mathrm{Pl}}$, and the effective heat capacity $\mathbb{C}_{V}=k_{\mathrm{B}}$. Then formula (15) for the effective temperature dispersion looks like

$(\Delta \mathbb{T})^{2}=\left(\mathbb{T}_{0}\right)^{2}$

and the relative dispersion of the effective temperature of this object obeys condition (3) now. It is the difference of this quantity from the dispersion of the Kelvin temperature of the same object in the quantum statistical mechanics. Accordingly, the relative dispersion of its internal energy, with regard for the general formula (17) and the formulas $C_{V}=k_{\mathrm{B}}$ and $\mathbb{U}=k_{\mathrm{B}} \mathbb{T}_{0}$, reads

$$
\frac{(\Delta \mathbb{U})^{2}}{\mathbb{U}^{2}}=\frac{k_{\mathrm{B}} C_{V}\left(\mathbb{T}_{0}\right)^{2}}{\left(k_{\mathrm{B}} \mathbb{T}_{0}\right)^{2}}=1,
$$

and condition (2) is also satisfied.

To make a more detailed comparison between the formulas obtained here and those known from the quantum statistical mechanics, let us express the dispersion of the effective internal energy of a quantum-mechanical oscillator in the form

$(\Delta \mathbb{U})^{2}=\left(\frac{\hbar \omega}{2}\right)^{2}\left[1+\sinh ^{-2}\left(\varkappa \frac{\omega}{T_{0}}\right)\right]$.

By comparing formulas (19) and (8), where the heat capacity looks like expression (7), we can present the second term in Eq. (19) in the form that reminds the initial formula (5), but includes an explicit dependence on the Kelvin temperature $T_{0}$,

$(\Delta \mathbb{U})^{2}=\left(\frac{\hbar \omega}{2}\right)^{2}+k_{\mathrm{B}}\left(C_{V}\right)_{\mathrm{qu}} T_{0}^{2}$.

Expression (20) evidently differs from expression (9) by an additional term, which can be written down in the form

$$
\left(\frac{\hbar \omega}{2}\right)^{2}=\frac{\hbar}{2} \rho_{\omega}(\omega, 0) \omega^{2},
$$

2 Note that the scope of problems, which can be solved with the use of this model, is wide enough, because the potential energy can be approximated by a parabola in a vicinity of its minimum. 
$\left.\rho_{\omega}(\omega, 0) \equiv \frac{\partial \mathbb{U}}{\partial \omega}\right|_{T=0}=\frac{\hbar}{2}$

is the spectral density of the effective internal energy at $T_{0}=0$. Then, formula $(20)$ reads

$(\Delta \mathbb{U})^{2}=\frac{\hbar}{2} \rho_{\omega}(\omega, 0) \omega^{2}+k_{\mathrm{B}}\left(C_{V}\right)_{\mathrm{qu}} T_{0}^{2}$.

It is worth to note that, in contrast to the quantumstatistical formula (8) valid for quantum-mechanical oscillators, formula (23) of the developed theory contains an additional term, which reveals itself at $T_{0}=0$ as well. Really, in the limit $T_{0} \rightarrow 0$, the second term in formula (23) disappears, so that

$$
\left(\Delta \mathbb{U}^{\mathrm{min}}\right)^{2}=\frac{\hbar}{2} \rho_{\omega}(\omega, 0) \omega^{2}=\left(\mathbb{U}^{\mathrm{min}}\right)^{2}=\left(\frac{\hbar \omega}{2}\right)^{2} \neq 0 .
$$

In the quantum statistical mechanics, on the contrary, $\left(\Delta U_{\mathrm{qu}}\right)^{2} \rightarrow 0$ at $T_{0} \rightarrow 0$. Hence, in the theory developed here, the validity of condition (2) for the effective internal energy both at an arbitrary Kelvin temperature and at $T_{0} \rightarrow 0$ is essentially associated with the account for the energy of zero oscillations.

In our opinion, another important result was also obtained in this consideration. It consists in that the zero energy has not also an exact invariable value, but possesses a certain "dispersion" or "width", by fluctuating within its limits. Naturally, there arises a question: "At the expense of what does it take place?" The answer is that, in this case, the system is in the equilibrium contact with "cold" (in the Kelvin sense) vacuum, which renders a quantum-mechanical stochastic action. The latter results in the randomization of the internal energy of an object even at the absolute zero temperature.

\section{Fluctuations of Effective Entropy and Their Correlation with Fluctuations of Effective Temperature}

Under the thermal equilibrium conditions, besides the fluctuations of individual macroparameters, $\delta A$ and $\delta B$, a considerable role is also played by the correlation between them. The measure of this correlation is known to be given by the expression $\sigma_{A B} \equiv\langle\delta A, \delta B\rangle$ referred to in the general case as the correlator.

Let us analyze the correlation between the fluctuations of effective macroparameters. In so doing, we are interested in a nontrivial case of conjugate effective macroparameters. It is known that the concept of conjugate quantities is one of the key ones in quantum mechanics.
Nevertheless, it is also used in thermodynamics, but, as a rule, on the basis of heuristic considerations. While analyzing the thermodynamic potentials (see, e.g., work [13] by Sommerfeld), it hits you in the eye that they contain some stable combinations of macroparameters, such as $(A d a)$ or $(a d A)$. For instance, the temperature is always combined with the entropy, and the pressure with the volume. Physically, those relations reveal themselves in every pair in the existence of a nonzero correlator between macroparameter fluctuations, which reflects their mutual interdependence. This circumstance forms the initial base to consider the pair of effective quantities $\mathbb{S}$ and $\mathbb{T}$ as thermodynamically conjugate, like the pair coordinate-momentum in quantum mechanics.

To substantiate this statement within the developed theory, let us calculate the dispersion of the effective entropy and the correlator between the fluctuations of the effective entropy and the effective temperature. Taking into account that, at a fixed effective volume $\mathbb{V}$, the fluctuation of the effective entropy is

$\delta \mathbb{S}=\left.\frac{\delta \mathbb{U}}{\mathbb{T}_{0}}\right|_{\mathbb{V}}=\frac{1}{\mathbb{T}_{0}} \mathbb{C}_{\mathbb{V}} \cdot \delta \mathbb{T}$,

we obtain the following expression for the corresponding dispersion:

$(\Delta \mathbb{S})^{2} \equiv\left\langle(\delta \mathbb{S})^{2}\right\rangle=\frac{\mathbb{C}_{V}^{2}}{\mathbb{T}_{0}^{2}}\left\langle(\delta \mathbb{T})^{2}\right\rangle=\frac{\mathbb{C}_{V}^{2}}{\mathbb{T}_{0}^{2}}(\Delta \mathbb{T})^{2}$.

Taking into consideration the consequence of general formula (15) for the standard deviation of the effective temperature, $(\Delta \mathbb{T})=\left(k_{\mathrm{B}}\right)_{0}^{1 / 2} \mathbb{T}\left(\mathbb{C}_{\mathbb{V}}\right)^{-1 / 2}$, and extracting the square root of expression (26), we obtain that the standard deviation of the effective entropy reads

$\Delta \mathbb{S}=\frac{\mathbb{C}_{V}^{2}}{\mathbb{T}_{0}}(\Delta \mathbb{T})=\left(k_{\mathrm{B}} \mathbb{C}_{V}^{2}\right)^{1 / 2}$

To derive the required fluctuation correlator $\sigma_{\mathbb{S T}}$, let us use formula (25) once more. We obtain that this correlator is proportional to the temperature dispersion:

$\sigma_{\mathbb{S T}}=\langle\delta \mathbb{S} \cdot \delta \mathbb{T}\rangle=\frac{\mathbb{C}_{V}}{\mathbb{T}_{0}}\langle\delta \mathbb{T} \cdot \delta \mathbb{T}\rangle=\frac{\mathbb{C}_{V}}{\mathbb{T}_{0}}(\Delta \mathbb{T})^{2}$.

Taking Eq. (15) into account, the corresponding fluctuation correlator is

$\sigma_{\mathbb{S T}}=\frac{\mathbb{C}_{V}}{\mathbb{T}_{0}} \frac{k_{\mathrm{B}}}{\mathbb{C}_{V}}\left(\mathbb{T}_{0}\right)^{2}=k_{\mathrm{B}} \mathbb{T}_{0}$,

so that the dependence on the effective heat capacity disappears. In other words, the correlator of the effective macroparameters $\sigma_{\mathbb{S T}}$ is governed only by a parameter of the quantum thermostat, namely, by its effective 
temperature, which, in accordance with Eq. (12), does not equal zero in principle. Therefore, it behaves like a quantum-mechanical correlator between the variables $p$ and $q$ in quantum mechanics, which also does not equal zero. This circumstance comprises an additional argument to consider the effective macroparameters $\mathbb{S}$ and $\mathbb{T}$ as conjugate quantities.

\section{Interrelation between the Correlation of Conjugate Micro- and Macroparameter Fluctuations and the Effective Action by the Environment}

To elucidate the physical sense of the expression for $\sigma_{\mathbb{S T}}$ in the form $(29)$, we address to the $(\hbar, k)$-dynamics [8, 10]. In so doing, we proceed from Bogoliubov's idea [14], according to which only the stochastic action of the environment can invoke a nontrivial correlation between fluctuations of the micro- and macroparameters. In the framework of the $(\hbar, k)$-dynamics [10], it was found that, at the microlevel, this action is described by a specific operator, Schrödingerian,

$\hat{\jmath} \equiv \delta \hat{p} \cdot \delta \hat{q}=\hat{\sigma}-i \hat{\jmath}_{0}$,

where

$\delta \hat{p}=\hat{p}-\langle|\hat{p}|\rangle ; \quad \delta \hat{q}=\hat{q}-\langle|\hat{q}|\rangle ;$

$\hat{\sigma} \equiv \frac{1}{2}\{\delta \hat{p}, \delta \hat{q}\} ; \quad \hat{\jmath}_{0} \equiv \frac{i}{2}[\hat{p}, \hat{q}]=\frac{\hbar}{2} \hat{I}$,

and $\hat{I}$ is the unit operator.

As a natural measure for the stochastic action by the environment at the microlevel, we introduced a specific parameter, the action $\mathcal{J}$, defined as the absolute value of the averaged Schrödingerian,

$\mathcal{J} \equiv \sqrt{\Sigma^{2}+\frac{\hbar^{2}}{4}}$

where $\Sigma$ and $\frac{\hbar}{2}$ are the average values for the operators $\hat{\sigma}$ and $\hat{\jmath}_{0}$, respectively. The quantity

$\Sigma=\left|\left\langle\psi^{*}(q)|\hat{\sigma}| \psi(q)\right\rangle\right|=\frac{\hbar}{2} \alpha$

is determined by the phase of the complex wave function

$\psi(q)=\left[2 \pi(\Delta q)^{2}\right]^{-1 / 4} \exp \left\{-\frac{q^{2}}{4(\Delta q)^{2}}(1-i \alpha)\right\}$.

A similar form has, e.g., the wave function of a free microparticle, which describes a smearing of its initial state in time at the zero Kelvin temperature [15].
However, the meaning of $\mathcal{J}$ can be interpreted differently. It can also be regarded as the absolute value of "quantum-mechanical correlator" $|\langle\delta p \mid \delta q\rangle|$ for the fluctuations of canonically conjugate quantities, the coordinate and the momentum,

$\mathcal{J} \equiv|\langle|\hat{\jmath}|\rangle|=|\langle|\delta \hat{p} \cdot \delta \hat{q}|\rangle|=|\langle\delta p \mid \delta q\rangle|$

At the macrolevel and in the equilibrium state with the quantum thermostat, the applicability region for definition (32) becomes wider $[8,10,11]$. The role of the wave function used for averaging the operator $\hat{\jmath}$ is played by a complex-valued wave function of warm vacuum in the coordinate representation, which depends on the Kelvin temperature of the quantum thermostat,

$\psi_{T_{0}}(q)=\left[2 \pi(\Delta \mathbb{Q})^{2}\right]^{-1 / 4} \exp \left\{-\frac{q^{2}}{4(\Delta \mathbb{Q})^{2}}\left(1-i \alpha_{T_{0}}\right)\right\}$,

where the parameter

$\alpha_{T_{0}}=\frac{1}{\sinh \left(\varkappa \frac{\omega}{T_{0}}\right)}$

is included into the wave-function phase, and

$(\Delta \mathbb{Q})^{2}=\frac{\hbar}{2 m \omega} \operatorname{coth}\left(\varkappa \frac{\omega}{T_{0}}\right)$

is the coordinate dispersion.

As a result, the quantity

$\mathbb{J}_{0}=\left|\left\langle\psi_{T_{0}}^{*}(q)|\hat{\jmath}| \psi_{T_{0}}(q)\right\rangle\right|=$

$=\sqrt{\frac{\hbar^{2}}{4} \alpha_{T_{0}}^{2}+\frac{\hbar^{2}}{4}}=\frac{\hbar}{2} \operatorname{coth}\left(\varkappa \frac{\omega}{T_{0}}\right)$,

which is equivalent to $\mathcal{J}$, appears as a natural characteristic of the integral stochastic action at the macrolevel. The quantity $\mathbb{J}_{0}$ was introduced earlier empirically as a new effective thermodynamic parameter, the effective action of the quantum thermostat, typical of stochastic thermodynamics $[9,11]$. Its important feature consists in that it is formally equivalent to other effective macroparameters of equilibrium stochastic thermodynamics and is genetically coupled with the microdescription through the wave-function phase depending on $\alpha_{T_{0}}$. This circumstance can be used for the interpretation of deeper interrelations between two levels of the description of the 
Nature, the quantum-mechanical theory and the thermodynamics. In particular, when comparing Eqs. (34) and (36), one can see that

$\Sigma_{T_{0}}=\left|\left\langle\psi_{T_{0}}^{*}(q)|\hat{\sigma}| \psi_{T_{0}}(q)\right\rangle\right|=\frac{\hbar}{2} \alpha_{T_{0}}$.

At the same time, using formula (7) for the heat capacity $\left(C_{V}\right)_{\mathrm{qu}}$, we obtain

$\Sigma_{T_{0}}=\frac{T_{0}}{\omega} \sqrt{k_{\mathrm{B}}\left(C_{V}\right)_{\mathrm{qu}}}$.

Hence, the same quantity $\Sigma_{T_{0}}$ characterizing the thermal action in the equilibrium state is strictly coupled with a nonzero phase of the warm-vacuum wave function at the microlevel and, simultaneously, with a nonzero heat capacity at the macrolevel.

In work [8], it was shown that, in the state of equilibrium between a quantum-mechanical oscillator and the quantum thermostat, the quantum-mechanical correlator "coordinate--momentum" looks like

$\sigma_{\mathbb{P Q}}=\frac{\hbar}{2} \operatorname{coth}\left(\varkappa \frac{\omega}{T_{0}}\right)=\mathbb{J}_{0}$,

We emphasize that, in the given context, the quantities $p$ and $q$ acquire the meanings of the effective macroparameters $\mathbb{P}$ and $\mathbb{Q}$ of the object in the model of quantummechanical oscillator, which is in the state of equilibrium with the environment.

Changing over to the microdescription in the limit $T_{0} \rightarrow 0$, formula (39) can be transformed to the form

$\sigma_{\mathbb{P Q}}^{\min }=\mathbb{J}_{0}^{\min }=\frac{\hbar}{2}$,

where the minimum value of $\sigma_{\mathbb{P Q}}$ is determined by Planck's constant that characterizes the quantummechanical stochastic action. A comparison of formulas (40) and (36) suggests that, in the general case, the effective action $\mathbb{J}_{0}$ in the equilibrium state can be presented in the form

$\mathbb{J}_{0}=\frac{1}{2} \hbar^{*}\left(\hbar, k_{\mathrm{B}}\right) \geqslant \frac{\hbar}{2}$,

where the quantity $\hbar^{*} \equiv \hbar \operatorname{coth}\left(\frac{\hbar \omega}{2 k_{\mathrm{B}} T_{0}}\right)$ can be naturally interpreted as a generalization of the elementary action $\hbar$ to the case with $T_{0} \neq 0$.

While comparing formulas (12) and (36), one can see that the quantities $\mathbb{J}_{0}$ and $\mathbb{T}_{0}$ are proportional to each other,

$\mathbb{T}_{0}=\frac{\omega}{k_{\mathrm{B}}} \mathbb{J}_{0}$
This fact gives grounds to use $\mathbb{J}_{0}$, rather than $\mathbb{T}_{0}$, as a "marker" of the equilibrium with the environment in all thermodynamic relations.

We now consider the correlator of fluctuations of the conjugate macroparameters, the effective entropy and the effective temperature, typical of the macrodescription. Using interrelation (42), we demonstrate that the correlator $\sigma_{\mathbb{S T}}$ of the form (29) also depends on $\mathbb{J}_{0}$. Really, in view of formula (42), relation (29) can be presented in the form

$\sigma_{\mathbb{S T}}=\langle\delta \mathbb{S}, \delta \mathbb{T}\rangle=\omega \mathbb{J}_{0}$.

Examining the limiting (at the Kelvin temperature $T_{0} \rightarrow$ $0)$ correlator value $\sigma_{\mathbb{S T}}=\omega \mathbb{J}_{0}^{\mathrm{min}}$, we see that, in this case, it is determined only by a stochastic action of the quantum-mechanical type,

$\sigma_{\mathbb{S T}}^{\min }=\omega \mathbb{J}_{0}^{\min }=\mathbb{U}_{0}^{\min }=\frac{\hbar \omega}{2}$,

where the energy of zero oscillations stands on the right.

Hence, according to formulas (40) and (44), the minimum values of fluctuation correlators for both pairs of the conjugate variables, $(\mathbb{P}, \mathbb{Q})$ and $(\mathbb{S}, \mathbb{T})$, are determined by the same Planck's constant. As the temperature grows, the correlators $\sigma_{\mathbb{P Q}}$ and $\sigma_{\mathbb{S T}}$ increase synchronously, remaining proportional to each other.

\section{Schrödinger uncertainty relations and their role in the theory of fluctuations of micro- and macroparameters}

It is known that the physical content of the uncertainties relation (UR), introduced for the first time by $\mathrm{W}$. Heisenberg in quantum mechanics, has been associated for many years exclusively with the theory of measurements. However, it was found that URs take place in other theories as well: in the equilibrium thermodynamics, theory of Brownian motion, and so forth. In our opinion, this circumstance is related to the stochastic action of an environment, which implicitly generates URs in those theories. From this viewpoint, a search for a universal relation between URs arising at zero and finite temperatures is quite justified. The analysis of fluctuations of conjugate micro- and macroparameters, which was carried out above, allows URs to be interpreted in the framework of the fluctuation theory developed here.

From the mathematical viewpoint, the most general UR proposed by E. Schrödinger (SUR) is an implementation of the Cauchy-Buniakowski-Schwarz inequality in the space of corresponding quantities [15],

$\mathcal{U} \mathcal{P}_{A B} \equiv \Delta A \Delta B \geqslant \sigma_{A B}$. 
Here, $\mathcal{U P}_{A B}$ is the product of standard derivations (the "uncertainties product"). The standard deviations $\Delta A$ and $\Delta B$ play the role of a measure for the "uncertainties" of random variables, the macroparameters $A$ and $B$. Accordingly, $\sigma_{A B}$ is the correlator of fluctuations of those quantities.

For conjugate micro- and macroparameters, the nonzero correlator of fluctuations imposes a restriction on the mutual behavior of standard deviations of those quantities. To show the fundamental character of similar restrictions, the left- and right-hand sides in relation (45) should be calculated independently, both in the microand macro-theories.

To calculate the quantity $\mathcal{U} \mathcal{P}_{\mathbb{S T}}$, which is typical of the macrotheory, we use formulas (27) and (15). As a result, we obtain that this quantity does not depend on the effective heat capacity and looks like

$\mathcal{U} \mathcal{P}_{\mathbb{S T}} \equiv(\Delta \mathbb{S})(\Delta \mathbb{T})=k_{\mathrm{B}} \mathbb{T}_{0}$

Comparing formulas (46) and (29), we notice that, in the state of equilibrium between the object, which is simulated as a quantum-mechanical oscillator, and the quantum thermostat, two different physical quantities, $\mathcal{U} \mathcal{P}_{\mathbb{S T}}$ and $\sigma_{\mathbb{S T}}$, have identical values. Therefore, the SUR "effective entropy-effective temperature" becomes an equality in this case, i.e. it is saturated.

$\mathcal{U} \mathcal{P}_{\mathbb{S T}}=\sigma_{\mathbb{S T}}$.

If $\mathbb{V} \neq$ const owing to the growing $\Delta \mathbb{S}$ at a immutable $\sigma_{\mathbb{S T}}$, the discussed SUR is transformed into an inequality, so that, in the general case, we have

$\mathcal{U} \mathcal{P}_{\mathbb{S T}} \geqslant \sigma_{\mathbb{S T}}$.

Now, let us take into account that the correlator $\sigma_{\mathbb{S T}}$ can be presented in the form (43). It is equivalent to the statement that mutual restrictions on the uncertainties $\Delta \mathbb{S}$ and $\Delta \mathbb{T}$ for an object in the equilibrium state are dictated by the integral stochastic action of the environment, which is characterized by the quantity $\mathbb{J}_{0}$. Therefore, in the equilibrium state, the SUR "effective entropy-effective temperature" takes the form

$\mathcal{U} \mathcal{P}_{\mathbb{S T}}=\omega \mathbb{J}_{0}=\omega \frac{\hbar^{*}}{2}=U_{\mathrm{Pl}}$

We should emphasize that the left- and right-hand sides of this SUR were obtained in the framework of the macrotheory, in which the expression for the Planck energy $U_{\mathrm{Pl}}$ is taken from the experiment. In the limit $T_{0} \rightarrow 0$, relation (49) looks like

$\mathcal{U} \mathcal{P}_{\mathbb{S T}}^{\min }=\omega \mathbb{J}_{0}^{\min }=U_{\mathrm{Pl}}^{\min }=\frac{\hbar \omega}{2}$, where the energy of zero oscillations stands on the right.

To calculate the quantity $\mathcal{U} \mathcal{P}_{\mathbb{P Q}}$ typical of the microtheory in the case of a quantum-mechanical oscillator, i.e., in the state of equilibrium with a quantummechanical thermostat, we use the expressions

$\Delta \mathbb{P}=\sqrt{\frac{\hbar m \omega}{2} \operatorname{coth}\left(\varkappa \frac{\omega}{T_{0}}\right)}$,

$\Delta \mathbb{Q}=\sqrt{\frac{\hbar}{2 m \omega} \operatorname{coth}\left(\varkappa \frac{\omega}{T_{0}}\right)}$,

which are contained in the wave functions of warm vacuum in the momentum and coordinate representations, respectively. Then we obtain

$\mathcal{U} \mathcal{P}_{\mathbb{P} \mathbb{Q}}=\frac{\hbar}{2} \operatorname{coth}\left(\varkappa \frac{\omega}{T_{0}}\right)$.

Comparing formulas (51) and (36), we obtain the SUR "coordinate-momentum" for a quantum-mechanical oscillator in the state of equilibrium with the quantum thermostat,

$\mathcal{U} \mathcal{P}_{\mathbb{P Q}}=\mathbb{J}_{0}$

with the minimum value $\mathbb{J}_{0}^{\min }=\hbar / 2$.

Note that, in contrast to SUR (49), both the leftand right-hand sides in SUR (52) were derived in the framework of the microtheory. The saturated form of this SUR is associated with the fact that the averaging of the corresponding operators of stochastic action was carried out with the use of the wave function depending on the temperature. The fact that both SURs (49) and (52) are governed by the same macroparameter, $\mathbb{J}_{0}$, is a substantial confirmation of the overlapping of those theories, which are traditionally referred exclusively to either micro- or macrodescriptions of the Nature.

It is worth demonstrating an interesting consequence of such an overlapping. It is known that the restriction imposed on the value of $\mathcal{U} \mathcal{P}_{\mathbb{P Q}}$ from below, which is equal to $\hbar / 2$, allows one to introduce, in the framework of the microtheory, elementary cells $2 \pi \hbar$ in dimensions in the "coordinate--momentum" phase space, which is a basis of the quasiclassical approximation in the quantummechanical theory. Following the analogy found above, it is also possible to introduce the "effective entropyeffective temperature" phase space in the framework of the macrotheory. The restriction on $\mathcal{U} \mathcal{P}_{\mathbb{S T}}$ equal to $\frac{1}{2} \omega \hbar^{*}$ at an arbitrary temperature means that this space is also 
partitioned into elementary cells of $2 \pi \omega \hbar^{*}$, the dimensions of which, however, increases with the temperature.

In this connection, there emerges a tempting idea to treat the theory of fluctuations of macroparameters in the framework of equilibrium stochastic thermodynamics as a quasiclassical theory in the phase space of the variables $(\mathbb{S}, \mathbb{T})$. In its turn, the quasiclassical approximation in the quantum-mechanical theory can be treated as a theory of fluctuations of microparameters in the phase space of the variables $(p, q)$. Such an interpretation allows the mathematical apparatus of the corresponding theories to be combined in the framework of a synthetic theory, which would substantially cover both the traditional thermodynamics and the traditional quantum-mechanical theory.

\section{Conclusions}

To summarize, we have proposed an approach, which allows the main paradox of the standard theory of fluctuations of macroparameters to be overcome. The paradox is associated with the fact that the conventional method of taking the quantum-mechanical effects into account leads to that the results obtained go beyond the scope of the thermodynamic description. As a result, on the basis of the quantum-mechanical model of thermostat and the microdescription within the $(\hbar, k)$ dynamics, a theory of quantum-thermal fluctuations of effective macroparameters and their correlation has been developed, with the corresponding thermodynamic description being preserved.

It has been demonstrated that the effective action, which is regarded as a macroparameter that reflects the stochastic influence of the environment, is responsible for the formation of corresponding dispersions and correlators. We also found that the uncertainties product of conjugate macroparameters, the effective entropy and the effective temperature, which characterizes the area of an elementary cell in the phase space, is confined from below by the energy of zero oscillations when approaching the absolute zero temperature.

In addition, we have showed that the correlators of the pairs of conjugate effective macroparameters $(\mathbb{S}, \mathbb{T})$ and $(\mathbb{P}, \mathbb{Q})$ in the equilibrium state are proportional to each other at any Kelvin temperature and linearly depend on the same macroparameter, the effective action $\mathbb{J}_{0}$ of the quantum thermostat.

At last, we have demonstrated that the minimum values of $\mathcal{U} \mathcal{P}_{\mathbb{P Q}}$ and $\mathcal{U} \mathcal{P}_{\mathbb{S T}}$ are determined by the same world constant; it is Planck's constant. It allows the theory of fluctuations of effective macroparameters to be regarded as a quasiclassical theory in the phase space of the variables $(\mathbb{S}, \mathbb{T})$, and the quasiclassical theory in the phase space of the variables $(p, q)$ as a theory of microparameters fluctuations.

We consider as a pleasant duty to express our gratitude to A.G. Zagorodny and Yu.P. Rybakov for their attention to the organization of seminars at the M.M. Bogoliubov Institute for Theoretical Physics of the NAS of Ukraine (Kyiv) and at the Department of theoretical physics of the Peoples' Friendship University of Russia (Moscow), respectively, as well as the participants at those seminars for the useful discussions. The work was sponsored by the Russian Foundation for Basic Research (project N 10-01-90408).

1. A. Einstein, in Collection of Scientific Works, edited by Ya.A. Smorodinskii (Nauka, Moscow, 1966), v. 3, p. 67 (in Russian).

2. L.D. Landau and E.M. Lifshitz, Statistical Physics, Part 1 (Pergamon Press, Oxford, 1980).

3. A.I. Anselm, Fundamentals of Statistical Physics and Thermodynamics (Nauka, Moscow, 1973) (in Russian).

4. A.D. Sukhanov, Phys. Particles, Nuclei 36, 1281 (2005).

5. A.D. Sukhanov and Yu.G. Rudoi, Phys.-Usp. 176, 551 (2006).

6. N.N. Bogoliubov, in Collection of Scientific Works, Vol. 6, edited by A.D. Sukhanov (Nauka, Moscow, 2006), p. 236 (in Russian).

7. A. Einstein, in Collection of Scientific Works, edited by Ya.A. Smorodinskii (Nauka, Moscow, 1966), v. 3, p. 145 (in Russian).

8. A.D. Sukhanov, JTMP 148, 1123 (2006).

9. A.D. Sukhanov, JTMP 154, 153 (2008).

10. A.D. Sukhanov and O.N. Golubeva, JTMP 160, 369 (2009).

11. A.D. Sukhanov and O.N. Golubeva, in Thermodynamics, edited by. T. Mizutani (InTech, Vienna, 2011), Ch. 4.

12. J.W. Gibbs, Elementary Principles of Statistical Mechanics (Dover, N.-Y., 1960). Ch. 9.

13. A. Sommerfeld, Thermodynamics and Statistical Mechanics (Academic Press, New York, 1956).

14. N.N. Bogoliubov, in Collection of Scientific Works, Vol. 6, edited by A.D. Sukhanov (Nauka, Moscow, 2006), p. 432 (in Russian).

15. A.D. Sukhanov, JTMP 132, 1276 (2002).

Received 05.07.11.

Translated from Russian by O.I. Voitenko 


\section{КВАНТОВО-ТЕПЛОВІ ФЛУКТУАЦІЇ ЕФЕКТИВНИХ МАКРОПАРАМЕТРІВ ТА ЇХ КОРЕЛЯЦІї}

\section{О.Д. Суханов, О.Н. Голубєва, В.Г. Бар'яхтар}

$\mathrm{P}$ е $з$ ю м е

Показано,що використання стандартної теорї флуктуацій макропараметрів удеяких випадках приводить до виходу за межі термодинамічного опису. Сформульовано основи теорії квантово-теплових флуктуацій ефективних макропараметрів та їх кореляції, узгодженої з умовами застосовності рівнова- жної термодинаміки і заснованої на ефективних макропараметрах, що враховують цілісний стохастичний вплив оточення за будь-яких температур. Обчислено корелятор спряжених макропараметрів - ефективної ентропії та ефективної температури - і встановлено його пропорційність ефективному впливу, що характеризує стохастичне оточення. Продемонстровано, що корелятори пар спряжених ефективних параметрів “ентропіятемпература" і "координата-імпульс" лінійно залежать від ефективного впливу, а їх мінімальні значення визначаються сталою Планка. 